\title{
VEGETATIVE NUCLEAR DIVISION IN NEUROSPORA
}

\author{
A. N. NAMBOodiri² AND R. J. Lowry \\ Department of Botany, University of Michigan, Ann Arbor
}

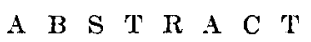

\begin{abstract}
A re-examination of the mode of vegetative nuclear division in Neurospora crassa was facilitated by the availability of the mutant "clock" which produces definite growth bands. Since the growth rhythm is correlated with nuclear divisions, stained mycelial mats of this mutant prepared at intervals from the beginning of a growth period provided a sequence of stages of division. In a 28-hour period the following broad features of nuclear behavior were observed: In the early part of the period during rapid mycelial growth, dividing elongated nuclei predominated. At the end of the period the mycelium contained mostly rounded resting nuclei. In the middle of a growth period nuclear forms of various degrees of annularity occurred along with elongated and rounded nuclei. Elongated and rounded nuclei completed division cycles without change in form, although the corresponding stages of the two types were similar. Elongated nuclei assumed a spiral form at the beginning of division. As division proceeded, relaxation of the nuclear gyres was accompanied by a visible duplication of the chromatin thread and the appearance of chromomere-like bodies on the daughter threads. One of the chromomere-like bodies became displaced and was interpreted to be a chromosome or a segment of a chromosome that acts as a mitotic center. All the chromosomes were found to be interconnected and to act as a unit throughout the division cycle. Only after the separation of the daughter chromatin threads could seven chromosomes be counted. Electron microscopic studies complemented the observations with the light microscope. On the basis of the evidence it was concluded that the vegetative nuclear division in Neurospora differs from the classical mitotic pattern in the following respects: (1) absence of visible centrioles, (2) the presence of interconnected chromosomes, (3) the comparatively late appearance of countable chromosomes, and (4) the frequent presence of interzonal connections between separating chromatin threads.
\end{abstract}

The manner of vegetative nuclear division in Neurospora is still a matter of controversy. Among others, Somers, Wagner, and Hsu (1960) and Ward and Ciurysek (1962) suggest that the nuclear divisions in the mycelium follow the classical mitotic pattern, whereas Bakerspigel (1959), Dowding and Weijer $(1960,1962)$ and Weijer, Koopmans, and Weijer (1963) observed stages that were not typical of mitosis. All these authors described the appearance of chromosomes at some stage, but there is very little agreement as to the structure of the nucleus or its exact mode of division.

A re-examination of nuclear division seemed to be appropriate with the isolation of the "clock" mutants (Sussman, Lowry, and Durkee, 1964). It was felt that the rhythmic growth of this mutant might be correlated with concomitant nuclear divisions. If so, "clock" mutants would offer the possibility of obtaining a sequence of

${ }^{1}$ Received for publication 4 August 1966.

This paper represents a portion of a dissertation presented by the senior author to the Graduate School of the University of Michigan, Ann Arbor, in partial fulfillment of the requirement for the degree of Doctor of Philosophy (1964). The work was supported in part by Grant GM09887 from the National Institutes of Ifealth, U.S. Public Health Service.

${ }_{2}$ Present address: T. C. 88, Minchin Road, Jagathi, Trivandrum-14, Kerala, India. division stages in a large number of nuclei, thereby eliminating reliance on sporadic divisions for the study of this process. Therefore, in this study attention was focused on the nuclear behavior of "clock" mutants, and comparisons were made with standard strains. Light microscopic observations were supported and extended wherever possible by electron micıscopic and enzymatic studies. A report integrating the information from all these sources is presented herein.

Materials and METHods-The strains of Neurospora crassa used in this study included two standard ones, hereafter called wild types (691113a and 65-811A) ${ }^{3}$ and two "clock" mutants (CL12a and CL11a). ${ }^{3}$ The gross morphology of the wild and the "clock" strains is strikingly different. Briefly, all wild strains have a spreading type of mycelium showing pronounced apical dominance. In contrast to the continuous growth shown by wild strains, "clocks" produce bands formed by alternating dense and sparse regions of hyphal growth. The time taken to complete one band is called a growth period which, under the conditions of this study, was approximately $28 \mathrm{hr}$. A detailed comparison of the morphology and

${ }^{3}$ Available from Fungal Genetics Stock Center, Dartmouth College, Hanover, N.H. 
physiology of wild and "clock" strains is given by Sussman et al. (1964), and Sussman, Durkee, and Lowry (1965).

Nearly all of the light microscopic observations included in this report were made on whole mounts of mycelial mats grown on sheet cellophane or dialysis tubing. Dialysis tubing containing "minimal" liquid medium (Vogel, 1956) was suspended in $500 \mathrm{ml}$-wide-mouthed jars with screw caps, sterilized, inoculated and incubated at $26 \mathrm{C}$. After the fungus had grown to the desired stage (usually between 16 and $72 \mathrm{hr}$ after inoculation) mycelial mats of the required size were cut out and fixed.

In other instances, especially in the study of nuclear division and changes of nuclear form during a growth period in "clock," the mutant was grown on a cellophane diaphragm stretched between two glass rings $14 \mathrm{~cm}$ in diam and $2.5 \mathrm{~cm}$ deep. Triangular pieces of cellophane $(2 \times 1 \mathrm{~cm})$ were placed on one side of the diaphragm and $10 \mathrm{ml}$ of "complete" medium (Horowitz, 1947) were carefully poured on the other. After sterilization the center of the side opposite the medium was inoculated. As the mutant grew over the cellophane, pieces were removed. Samples were taken at intervals of $0.5,1$, or $2 \mathrm{hr}$ (Fig. 1). The cellophane strip with the adhering mycelium was then fixed and stained.

The Giemsa-HCl staining technique used was based on the methods of Hrushovetz (1956) and Ward and Ciurysek (1962) with the following modifications: Giemsa stain $(0.2 \mathrm{gm})$ was dissolved in $10 \mathrm{ml}$ of glycerine and kept in an oven $(60 \mathrm{C})$ for $4 \mathrm{hr}$. The stock solution was made by adding $10 \mathrm{ml}$ of methyl alcohol to this mixture. Three ml of the stock solution were dissolved in $25 \mathrm{ml}$ of phosphate buffer at $p \mathrm{H} 7$. The staining solution prepared in this way was used immediately. The mycelium was fixed in either acetic-alcohol $(1: 3)$ or in alcohol-acetic acid-lactic acid $(6: 1: 1)$. Glacial acetic acid, absolute alcohol and fullstrength lactic acid were used to make up these solutions. Hydrolysis was for $4 \mathrm{~min}$ at room temperature followed by $10 \mathrm{~min}$ at $60 \mathrm{C}$ in $1 \mathrm{~N} \mathrm{HCl}$. The mycelium was then washed several times in water, followed by buffer, and then transferred to a vial containing freshly prepared staining solution. The time of staining varied with the thickness of the mycelium and its age, but most often $10 \mathrm{~min}$ gave good results. If necessary, the mycelial mats were destained in a dilute solution of Giemsa $(0.5 \mathrm{ml}$ of stock solution in $100 \mathrm{ml}$ of distilled water). Mounts were made in Abopon (Valnor Corporation, Brooklyn, N.Y.).

In the enzyme studies, deoxyribonuclease was used in $\mathrm{Mg}^{++}$-gelatin buffer solutions prepared according to the method of Leuchtenberger (1958). Samples for enzyme treatment and controls consisted of adjacent pieces of the same mycelial mat of approximately the same size.

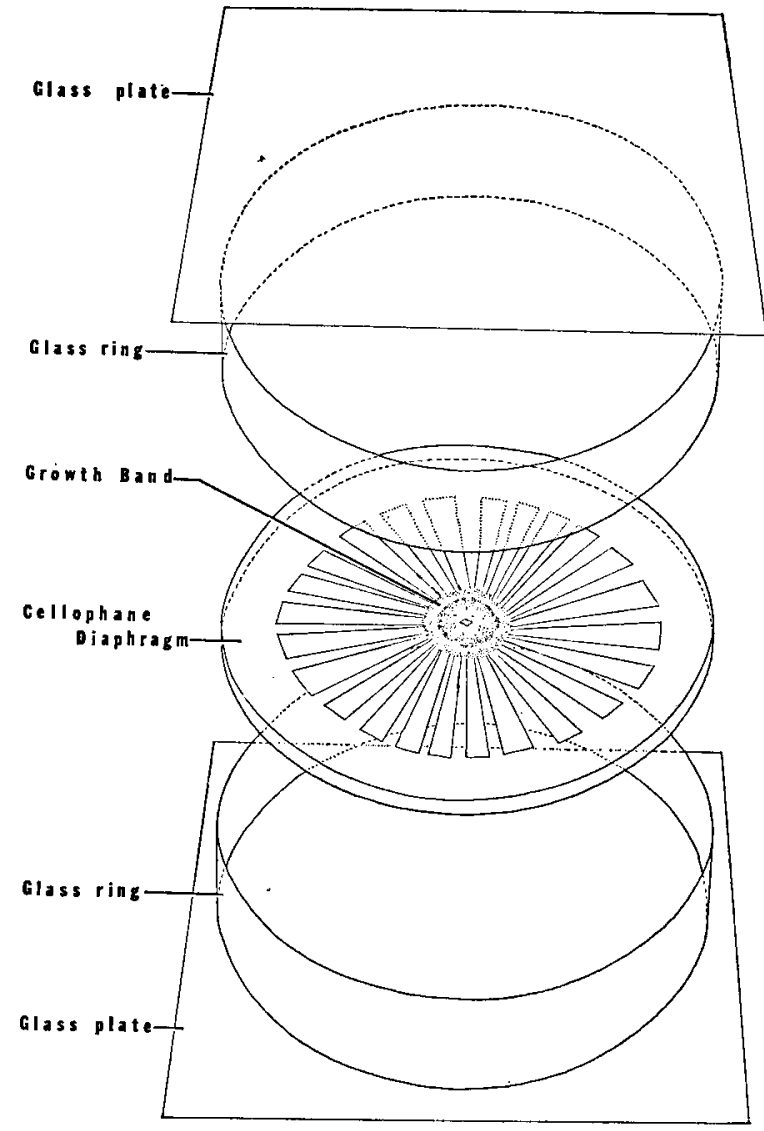

Fig. 1. Apparatus used for the culture of the "clock" mutants of Neurospora crassa.

Special care was taken to provide the same time of digestion, hydrolysis and staining for all samples.

In electron microscopy attention was focused on the beginning and end of a growth period in the "clock" mutants, where large numbers of dividing and resting nuclei, respectively, were seen under the light microscope. The mutant was cultured on dialysis tubing, and after the desired period of growth the mycelium was floated off in water. While still in water such mycelial pieces were layered to form a thick mat or, if the pieces were sufficiently large, folded over several times parallel to the direction of growth. Material so prepared was covered with melted $1 \%$ agar and when cooled to room temperature the excess of agar around the mycelium was removed. The mycelium embedded in agar was then divided into small cubes and fixed in $2 \%$ aqueous $\mathrm{KMnO}_{4}$ for $10-20$ $\min$.

The method of Luft (1961) was followed in embedding with epoxy resin. Sections were cut with glass knives with a Porter-Blum microtome and were floated onto uncoated 100-mesh copper grids and, in some cases, stabilized with a Pt-Pd coating. Observations were made with either a JEM-6A or RCA-3G electron microscope with 
an accelerating voltage of 80 , or $50 \mathrm{kv}$ respectively.

OBSERVATIONS - Numbers of nuclei in cellsPrevious reports on the number of nuclei per cell in Neurospora indicated the presence of several to many. For example, Bakerspigel (1959) counted at least 30 nuclei in one cell. By contrast the present observations showed that some cells, especially in younger hyphae, contained more than 100 stained bodies (Fig. 3). That these bodies are nuclei was confirmed by enzymatic digestions (Fig. 4-7). Moreover, the average number of nuclei per cell was the same after the following staining procedures: Feulgen, Giemsa, acetoorcein and aceto-iron-hematoxylin. Many nuclei also were found in thin sections observed in the electron microscope (Fig. 15, 18).

Diversity of nuclear forms-For the study of the occurrence and distribution of diverse forms of

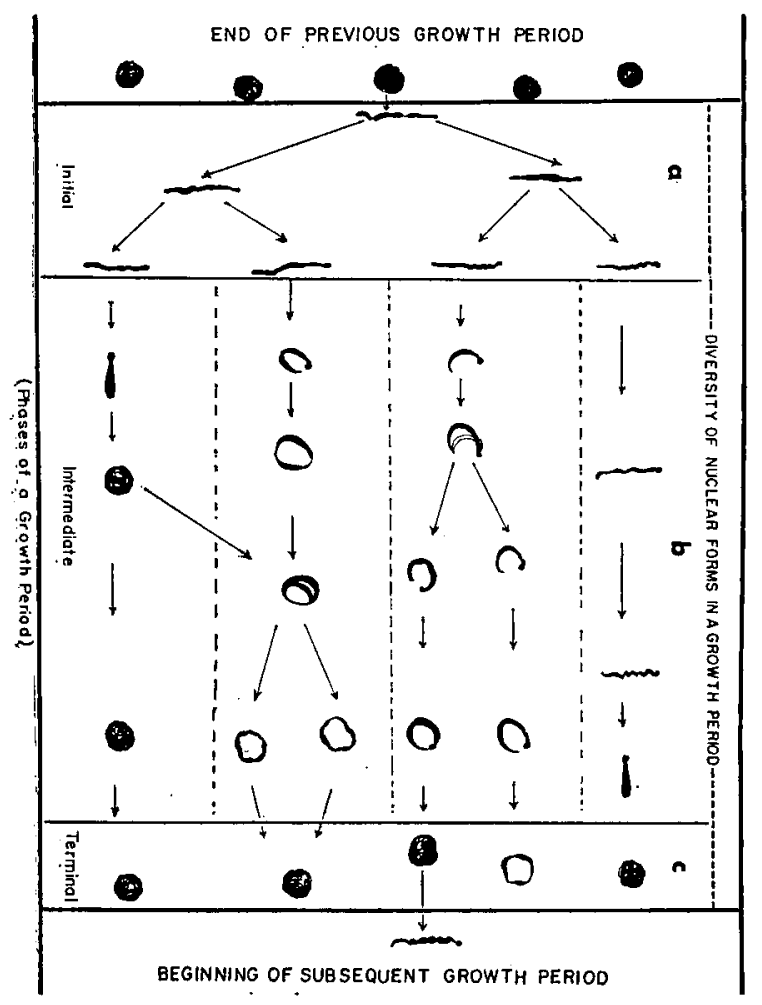

Fig. 2. Diagram showing the diversity of nuclear forms in a growth period of the "clock" mutant of Neurospora crassa.-a. Initial phase with predominantly elongated nuclei.-b. Intermediate phase. The elongated nuclei that enter this phase may: (1) continue to divide without change in form at a slower rate (first row), (2) change into incomplete ring nuclei and divide (second row), (3) transform into ring nuclei and divide (third row), (4) undergo longitudinal condensation to form rounded nuclei, some of which revert to ring nuclei and divide (fourth row).-c. Terminal phase with predominantly rounded nuclei. nuclei, a growth period may be divided into the initial, intermediate and terminal phases. The initial phase lasts for 4-10 hr. At the beginning of this phase the hyphae extend singly or in patches from the previous growth band and the nuclei that migrate into these hyphae become elongated (Fig. 2a, 10, 56). This condition persists throughout the initial phase. As the period progresses the branching of the initial hyphae as well as the grow th of new hyphae forms a continuous mycelial mat which marks the end of the initial phase. Mycelium during the intermediate phase contains elongated, rounded and ring nuclei, as well as nuclei of intermediate forms (Fig. 26, 58, 11-14). Inasmuch as elongated nuclei are found even in advanced stages of this phase, it is suggested that some of the elongated nuclei continue to divide and preserve their shape until the end of the growth period (Fig. 2b, row 1). Longitudinal condensation of elongated nuclei to form rounded ones (Fig. 20,45 lower pair) is suggested by our results (Fig. 2 b, row 4 ). The ring and incomplete ring nuclei are considered to be modifications of the elongated nuclei, since gradations from elongated forms with slightly curved ends through forms that show various degrees of annularity to complete ring nuclei are seen (Fig. 12, 14, 19-21, $37,51,69,52,70)$. The ring nuclei and intermediate forms between ring and elongated nuclei also divide without change during this phase (Fig. 12, 51, 69), but as growth progresses more and more of the nuclei become rounded (Fig. 2b, rows 2,3 ). As the period advances the general trend is toward an increase in the percentage of rounded nuclei. By the time the growth of the mycelium stops and the terminal phase lasting 2-3 hr begins most of the nuclei are in the rounded condition (Fig. 2c, 8, 9). With the beginning of the next period those nuclei that pass into the growing hyphae again transform into the elongate form.

Nuclear division-The study of the progress of division in "clock" strains was facilitated by a certain degree of correlation between growth and nuclear division as evidenced by the presence of elongated nuclei in the initial phase, rounded nuclei in the terminal, and a variety of nuclear forms in the intermediate phase of a growth period (Fig. 2). This correlation could not be extended to wild strains because of the absence of rhythmic growth, although there is a tendency for elongated nuclei to be more numerous in the young and active parts of the mycelium and rounded nuclei to predominate in the older and less actively growing regions. However, the various forms of nuclear division that were seen in wild strains were the same as those in "clock," and so it is suggested that nuclear division is similar in both types.

The elongated and rounded nuclei seen in all strains divide without losing their characteristic shape. Since most of the division stages detected 


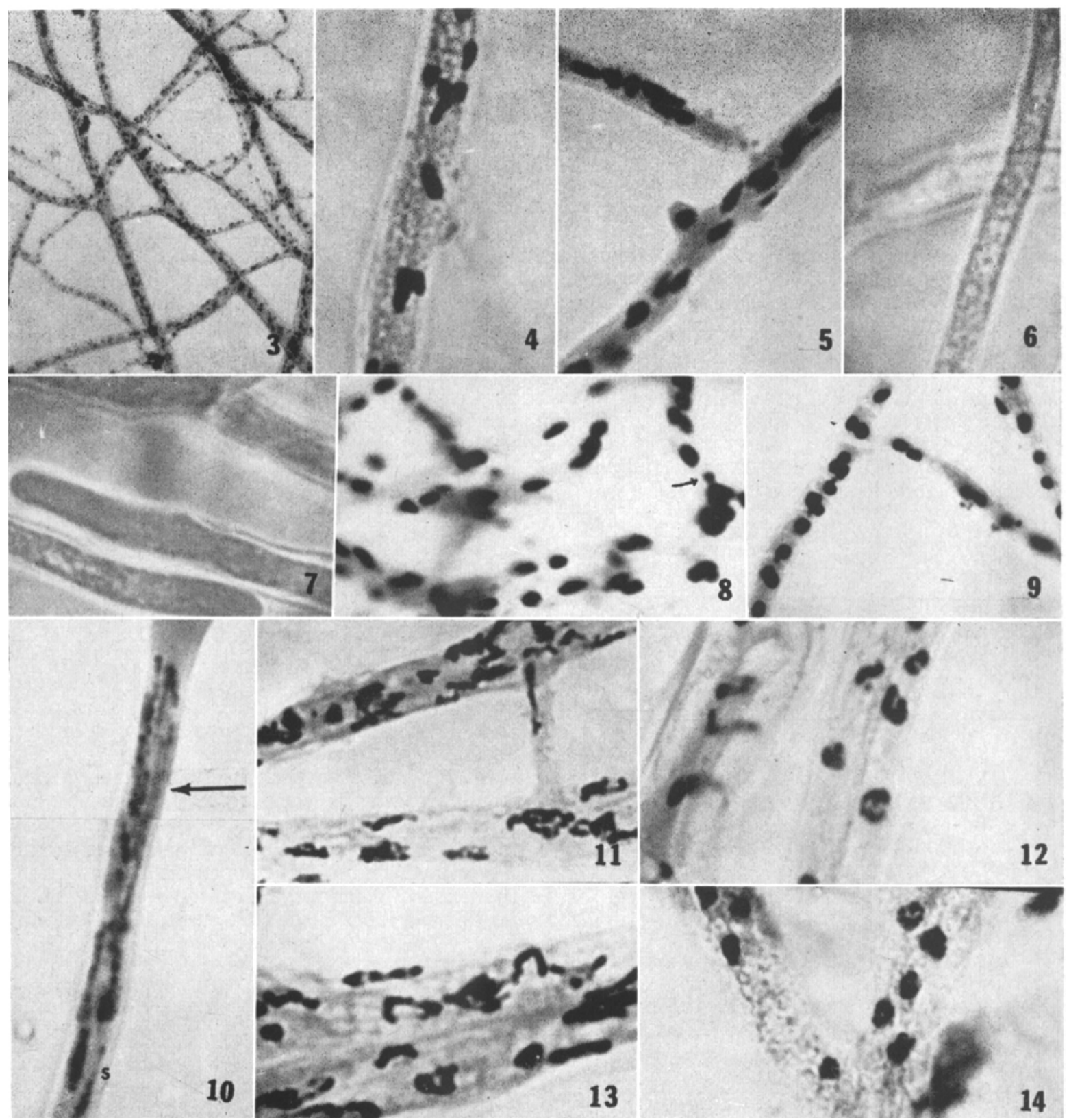

Fig. 3-14. The number and form of nuclei found in various growth phases of the "clock" mutant of Neurospora.Fig. 3. Giemsa-stained mycelial mat showing large numbers of closely packed nuclei in each cell. The average length of cells in this figure is $90 \mu, \times 300$.-Fig. 4. A portion of the hyphae from the terminal growth period used as controls for enzyme-digestion experiments. The stained structures are interpreted as resting nuclei, $\times 2000 .-$ Fig. 5. Hyphae from a portion of the mycelium adjacent to the control and treated in ribonuclease for $10 \mathrm{hr}, \times 2000$. - Fig. 6, 7. Deoxyribonuclease-treated hyphae. The background is the same as that of the control in Fig. 4, but stained bodies are absent here, $\times 2000$.-Fig. 8, 9. Predominance of rounded resting nuclei at the end of a growth period. Fig. 8 is an earlier stage in the end phase of a growth period. The arrow in this figure shows a late stage in the longitudinal condensation of the elongated nuclei to form rounded ones, $\times 800 .-$ Fig. 10. Elongated nuclei in the initial growth phase. The slender interchromosomal strand with which a knob-like structure is attached to the nucleus is indicated by the letter "s." The arrow shows a dividing nucleus with stretched daughter strands and to its left is a dividing nucleus with daughter strands wrapped around each other, $\times 2000$ (see Fig. 56). - Fig. 11-13. Adjacent parts from the mycelium in the early part of the intermediate growth phase. Elongated nuclei of various sizes are seen in Fig. 11.-Fig. 12, 13. Incomplete ring and rounded nuclei, $\times 2000$.-Fig. 14. Incomplete ring and ring nuclei from an advanced stage in the intermediate growth phase, $\times 2000$. 


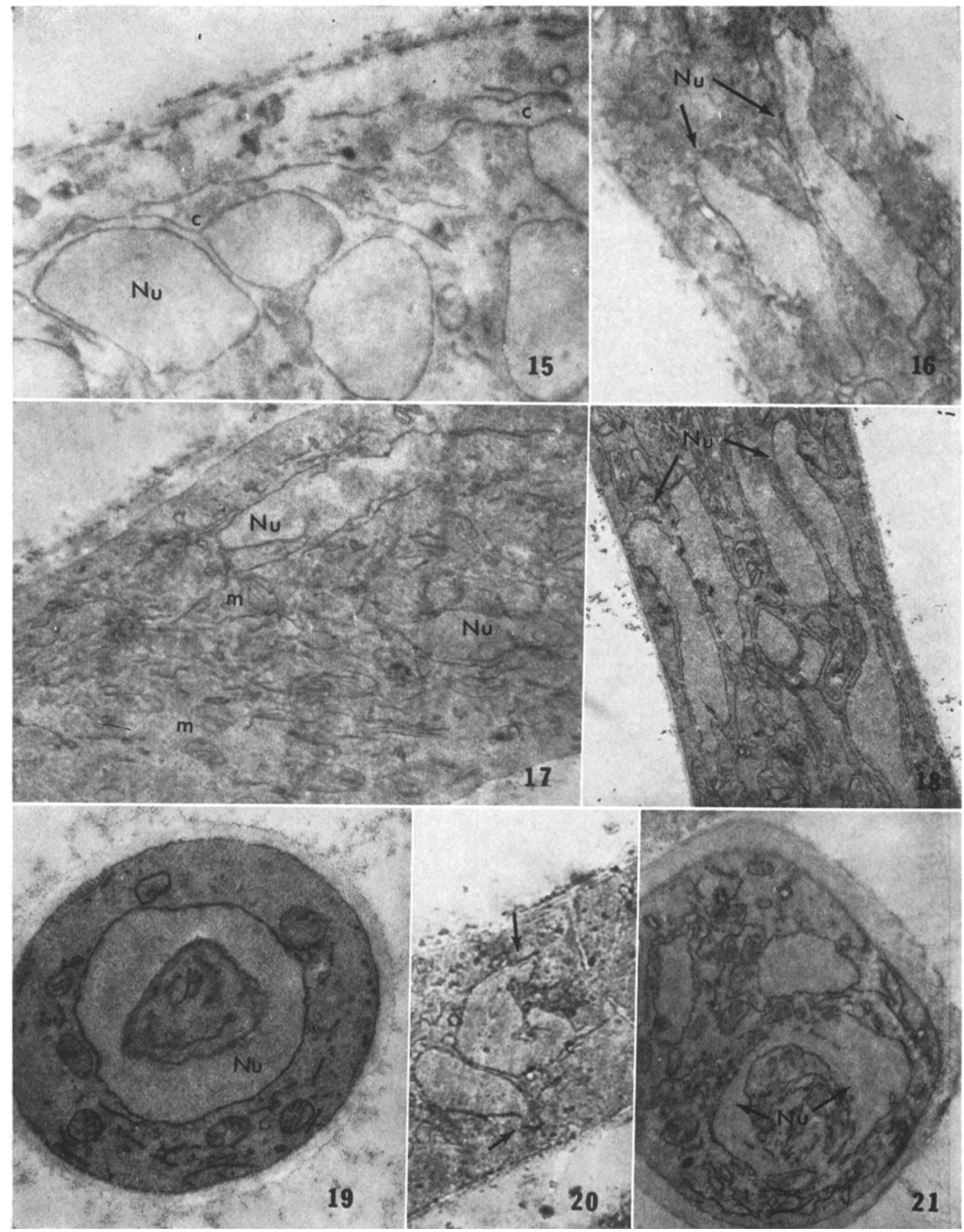

Fig. 15-21. Electron micrographs of thin sections of "clock" Neurospora hyphae.-Fig. 15. A hypha from the terminal growth phase showing crowded nuclei $(\mathrm{Nu})$ and continuity of the endoplasmic reticulum with the nuclear membrane is shown at $\mathrm{c}, \times 15,000$.-Fig. 16. Elongated nuclei $(\mathrm{Nu})$ at telophase, $\times 15,000 .-$ Fig. 17. Disintegration of nuclear envelope during the early stages of division (Nu-nucleus, m-mitochondrion), $\times 15,000$.-Fig. 18. Two pairs of daughter nuclei $(\mathrm{Nu})$ at the end of the division of two adjacent elongated nuclei, $\times 10,000 .-$ Fig. 19. Cross section of a hypha showing a ring nucleus (Nu), $\times 20,000$.-Fig. 20. An incomplete ring nucleus and a longitudinally condensing elongated nucleus. Both nuclei have knob-like structures (arrows), $\times 15,000$. - Fig. 21. Cross section of a hypha showing an incomplete ring nucleus $(\mathrm{Nu}), \times 12,500$. 


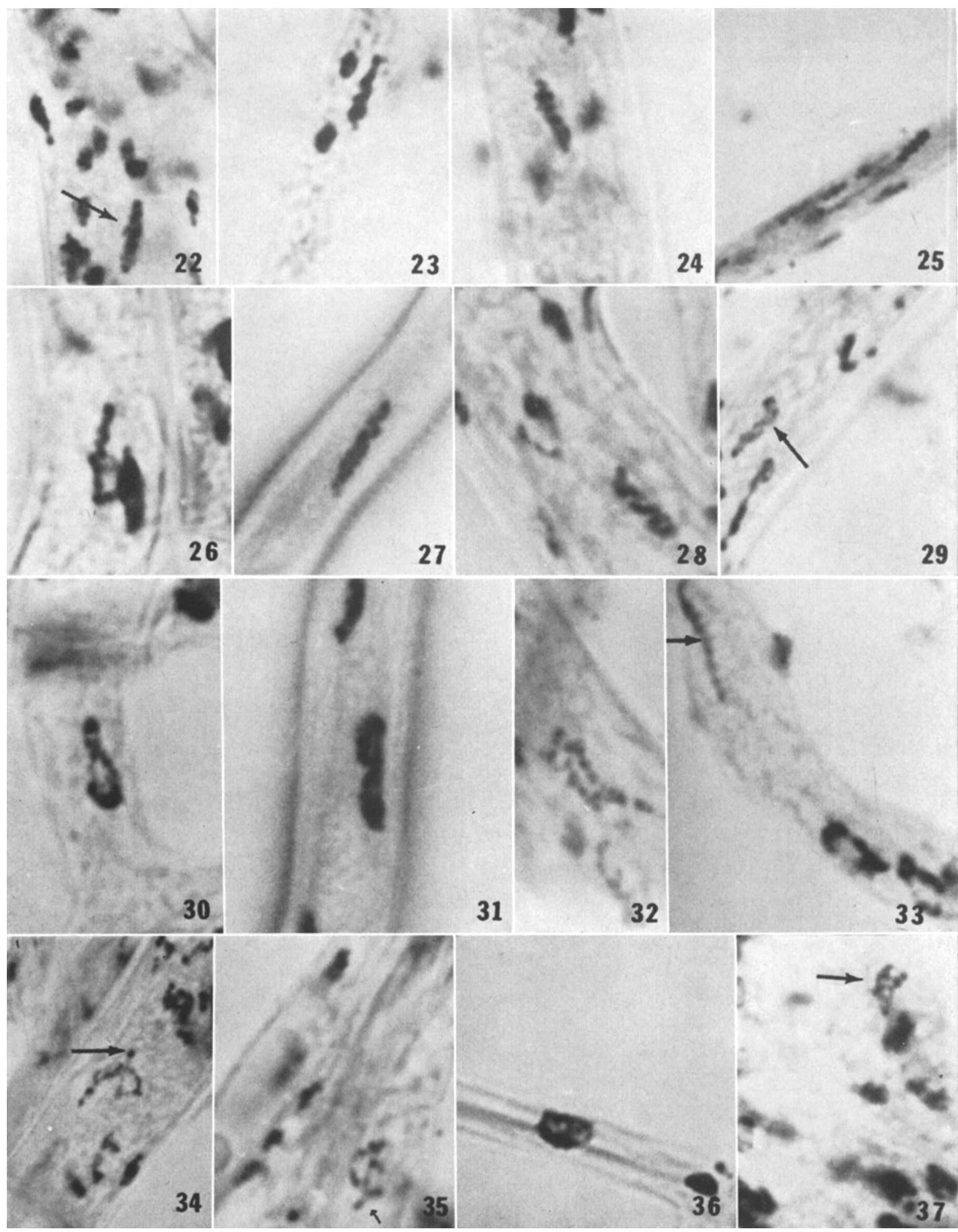

Fig. 22-37. Various stages of nuclear division in elongated nuclei of the "clock" mutant of Neurospora.-Fig. 22. Overstained elongated spiral nucleus (arrow). Most commonly spiral nuclei appear as corrugated structures since overstaining masks spirals.-Fig. 23. A spiral nucleus with the gyres to the right.-Fig. 24. A spiral nucleus with the gyres to the left.Fig. 25. A spiral nucleus in which the direction of the spiral reverses (see Fig. 57).- Fig. 26. A spiral nucleus immediately before division showing the beginning of despiralization (see Fig. 58).-Fig. 27. An elongated nucleus showing the dupli- 
were of elongated nuclei a description of their division is presented in detail, and this is followed by a comparison with the course of division in rounded forms.

Division of the elongated nuclei: InterphaseElongated nuclei under the electron microscope show distinct nuclear envelopes and occasional continuity of the envelope with endoplasmic reticulum (Fig. 16, 18). The distinct knob-like terminal portions of elongated nuclei seen in electron micrographs (Fig. 20, lower nucleus, arrow) are correlated with deeply stained portions of these nuclei observed under the light microscope (Fig. 45, lower pair of nuclei). The light micrographs also show that in some elongated dividing nuclei the knob-like portions undergo displacement but remain attached to the main body by thin weakly stained thread-like strands (Fig. 10, 56, 48s).

The elongated nuclei vary in length from 2-11 $\mu$ (Fig. 10, 56). Since an increase in the length of nuclei results in a proportional decrease in diameter, it is assumed that, due to forces of protoplasmic streaming or other reasons, the chromatinic material undergoes stretching after dissolution of the envelope.

Early stages of division: Appearance of coiled and duplicated forms-The elongated nuclei in the initial phase of a growth period appear straight, twisted or coiled (Fig. 10, 56). Coiled and duplicated forms predominate during the first $2 \mathrm{hr}$, but as the period progresses stages comparable to anaphase appear more and more frequently. Therefore, the coiled and duplicating nuclei that occur at the beginning of the period are considered to represent early stages of division.

Overstained undivided spiral nuclei often appear as corrugated structures (Fig. 22 arrow), but in properly differentiated preparations the coiled nuclei are seen to possess $3-5$ gyres (Fig. $23-26,57,58)$. The coils may be either righthanded (Fig. 23) or left-handed (Fig. 24) or, rarely, the same nucleus may be coiled to the left in one portion and to the right in the remainder (Fig. 25, 57).

Despiralization of the coiled nucleus (considered here to be a chromatinic strand) occurs in the later phases of the growth period, and this process is accompanied by the visible duplication of the nuclear material into two strands (Fig. 26, $58,27,59)$. In the majority of cases splitting begins from the end opposite the one with the darkly stained body or from the middle (Fig. 27, 59, 28, 29). The duplicating nuclear material may undergo stretching and may appear in some cases as narrow lightly staining threads (Fig. 10, 56, 44, arrows). In properly stained, unstretched, dividing nuclei, the daughter strands may or may not show a beaded appearance from the beginning of duplication (Fig. 27, 59, 32, 62, 33, 34, 63).

Ultrathin sections of the mycelium in the early growth period showed elongated nuclei with intact nuclear envelopes, but as the period advances the hyphae show only disintegrating membranes (Fig. 16). Some of the figures in electron micrographs can be correlated with images of dividing nuclei under lower magnification. This can be seen most clearly in a comparison of the parallel daughter nuclei at the end of division in electron micrographs (Fig. 16, 18) and light micrographs (Fig. 45-47).

Early stages of nuclear division are seen at high magnification in Fig. 17. The breakdown of the nuclear membrane is not associated with the disintegration of other cytoplasmic organelles like mitochondria. The lighter areas within the nuclear space are interpreted to be chromatinic material (Fig. 17).

Since specific DNA stains for electron microscopy are still being developed, the breakdown of the nuclear envelope makes it difficult to follow the course of nuclear division until the envelope is reassembled in a stage comparable to the telophase of classical mitosis (Fig. 16).

Separation of daughter chromatin-Intermediate stages in the process of separation of daughter strands are seen in certain cases under the light microscope. Separation of the strands may be effected readily in situations where, after duplication of the nucleus, the daughter strands lie parallel to each other (paranemic coiling) (Fig. 31, $61)$. Fig. 29 , however, represents a case where the daughter strands lie wrapped around each other (plectonemic coiling). Under such conditions the strands cannot separate along their entire length without rotation of their ends. Separation by this

cation of the chromatinic strands at one end. The individual strands show bead-like structures (see Fig. 59).-Fig. 28. A partially duplicated elongated nucleus showing duality in the duplicated region.-Fig. 29. Arrow shows an elongated nucleus where the separating strands are wrapped around each other.-Fig. 30. Despiralization of the strands (see Fig. 60).-Fig. 31. Separation of the strands without intertwining. Duplication proceeding from both ends (see Fig. 61).Fig. 32. A later stage in the separation of strands showing bead-like bodies (see Fig. 62).-Fig. 33. Arrow indicates a separated strand showing the beaded structure; the sister strand may have been removed by cytoplasmic streaming.Fig. 34. Separation of the strands displaced with one of the central chromosomes (arrow). The opposite displaced chromosome is out of focus (see Fig. 63).-Fig. 35. A configuration in which one of the displaced chromosomes (arrow) is a subterminal chromosome of the chain (see Fig. 64).-Fig. 36. Separation of the strands with non-parallel connections (see Fig. 65).-Fig. 37. Arrows show longitudinal duplication of the nuclei with parallel connections. All figures, $\times 2,000$. 


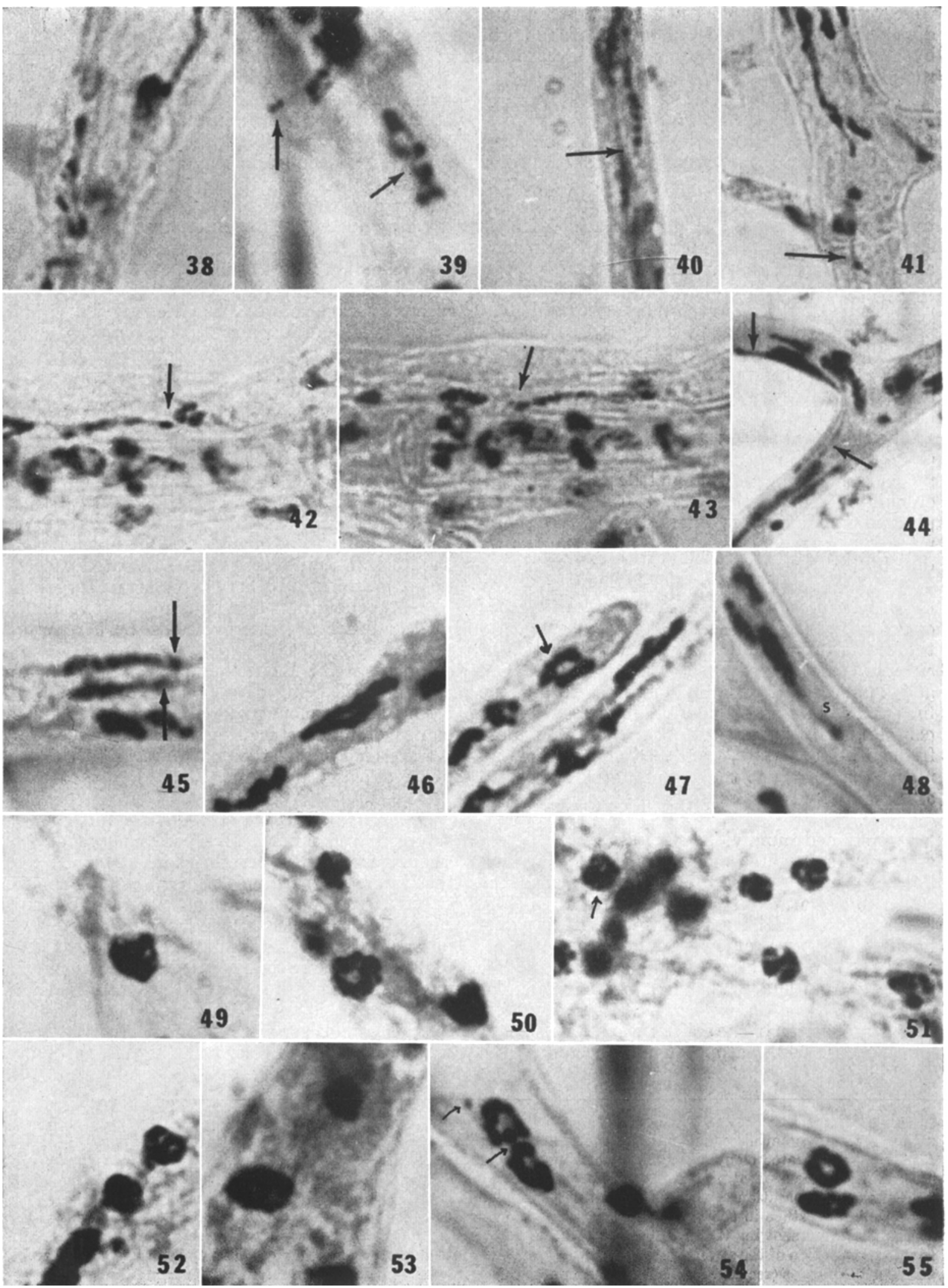


means is suggested by the dividing nucleus in Fig. 30,60 .

A variable number of bead-like bodies are seen on the daughter chromatinic strands in the earlier phases of division (Fig. 27, 59, 32, 62, 33b). With the separation of strands and progressive condensation of daughter strands two or more of these beaded bodies may merge to form chromosomes.

In some cases, before duplication is completed, a body that appears to be a chromosome is displaced from the beaded chain (Fig. 34, 63, arrow). A chromosome from a terminal or intermediate position in the chain may be displaced (Fig. 34, $63,35,64$, arrows) and the interchromosomal strand that connects the displaced chromosome may be seen in various stages of condensation (Fig. 38, 66, 39, 40, 67, 42). Therefore, it is suggested that the displaced chromosome acts as a focal point for other chromosomes to follow and thus it may act as a mitotic center.

Displaced chromosomes were not detected in all separating strands, especially where the strands show interconnections (Fig. 36, 65, 37). These connections may or may not be parallel to each other (Fig. 36, 65, 37, arrows). Interconnections between daughter strands also are seen in some of the dividing rounded nuclei (Fig. 50, 68, 51, 69 , arrow).

Time of appearance of chromosomes-Irrespective of the manner and mechanism of separation, chromosomes can be counted only after the separation of the daughter chromatin threads. The position of the displaced chromosome in the chain may determine the final shape of the chromosome configuration. For example, if the middle chromosome of a chain of seven chromosomes is displaced, the two arms of three chromosomes move toward each other to form an inverted Y-shaped structure (Fig. 38, 66, 39, lower arrow). If, however, the displaced chromosome is at one end of the chain, the chromosome configuration appears as a straight chain (Fig. 40, 67, 42, 43).

Interchromosomal strands. were present in most of the configurations studied. These strands are narrow, weakly stained and, depending upon the stage of division, variable in length (Fig. 38, $66,39)$.

Chromosome morphology-Seven chromosomes can be distinguished in some configurations. A group of six chromosomes with a seventh separated from the group is seen in Fig. 39, upper arrow. In other configurations one of the terminal chromosomes is darkly stained and a little displaced while the opposite chromosome has a satellite-like structure (Fig. 40, 67, 43). Rarely, it is this terminal chromosome with the satellite structure that is displaced (Fig. 41).

A chromosome group usually consists of two long, one medium and four short chromosomes. Variations in the length of chromatin strands due to stretching, with correlated changes in their diameter, might account for the differences in size and shape of chromosomes in different haploid complements.

Telophasic transformations-During the terminal phase of division the chromosomes become less distinct (Fig. 45). Electron micrographs of similar nuclei suggest that the nuclear envelope is reconstituted at this stage (Fig. 16). The upper pair of nuclei seen in Fig. 45 is considered to be similar to those in Fig. 16 where the nuclei are in telophase. The nuclear envelopes of daughter nuclei in Fig. 16 appear to be composed of fragments of membranes similar to those of the endoplasmic reticulum. The connection of the endoplasmic reticulum with the nuclear envelope also can be noticed (Fig. 15c). Under the light microscope displaced chromosomes are still distinguishable (Fig. 45) though in the final stages of nuclear reorganization the elongated daughter nuclei appear undifferentiated and undergo progressive condensation (Fig. 46, 47). From this stage the telophase nuclei may (1) pass to the next division cycle without reverting to the rounded form (Fig. 48), or (2) transform into

Fig. 38-55. Various stages of nuclear division in wild and "clock" strains of Neurospora.-Fig. 38. The distinct chromosome stage showing seven chromosomes with the central one displaced (see Fig. 66).-Fig. 39. Two chromosome groups; the lower consists of seven chromosomes (lower arrow) with their interchromosomal strands condensed, while in the upper a group of six chromosomes with one separated from the rest (upper arrow) is seen.-Fig. 40 . A chain of seven chromosomes with the terminal chromosome displaced. The chromosome at the opposite end of the chain has a satellitelike structure (arrow) (see Fig. 67).-Fig. 41. A displaced chromosome with a satellite.-Fig. 42, 43. A chromosome chain at two focal levels.- Fig. 42 shows the terminal chromosome which is displaced and heavily stained-Fig. 43 reveals the chromosome with the satellite.-Fig. 44. An elongated nucleus after the dissolution of the nuclear membrane with both ends stretched at the arrows.-Fig. 45. Two pairs of daughter nuclei undergoing telophasic transformation (arrows indicate displaced chromosomes). Two lower nuclei represent stages in the condensation of elongated nuclei to form rounded ones.-Fig. 46, 47. Telophase nuclei in the wild strain of Neurospora (arrow in Fig. 47 indicates the daughter telophasic nuclei).-Fig. 48. The beginning stage of a new division cycle ("s" is the strand that connects the main body of the nucleus with the knob-like portion).-Fig. 49-55. Division stages in rounded nuclei.-Fig. 49. A resting nucleus.Fig. 50. A ring stage with duplicated strands (see Fig. 68).-Fig. 51. Incomplete and complete ring nuclei (see Fig. 69).Fig. 52, 53. Duplicating ring nuclei, the daughter strands may or may not be interlocked (see Fig. 70, 71).-Fig. 54. Separation of daughter strands of interlocked rings. Arrows indicate the displaced chromosomes of the rings (see Fig.72). Fig. 55. Ring nuclei in telophase. All figures $\times 2,000$. 


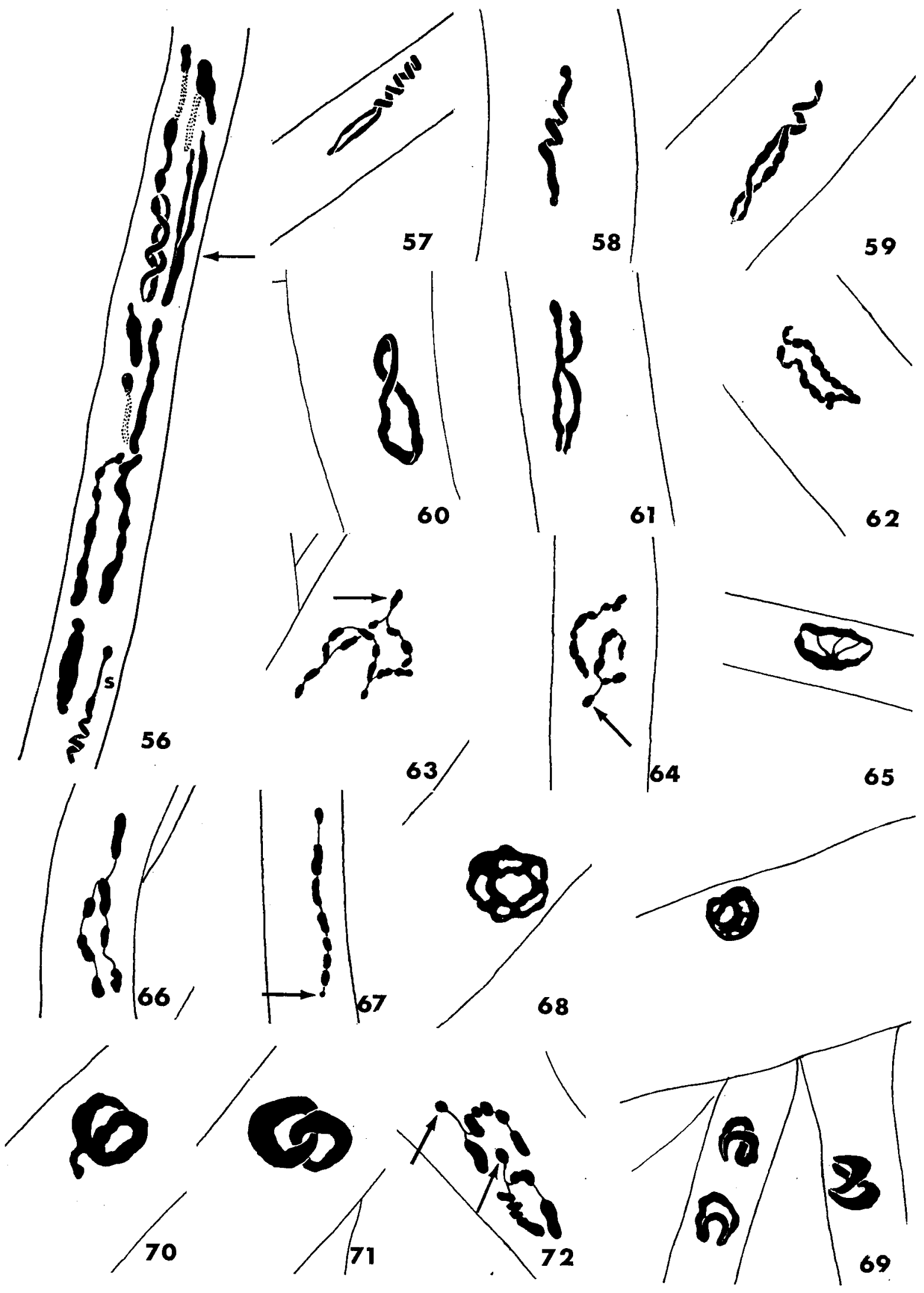


intermediate forms such as crescent, semi-ring and ring-shaped nuclei (Fig. 12, 14), or (3) undergo linear condensation to form rounded resting nuclei. A stage in the condensation of the elongated nuclei to rounded ones is shown in Fig. 45 (lower pair of nuclei).

Rounded nuclei: Interphase-The rounded nuclei seen in the terminal phase of a growth period (Fig. 8, 9) have distinct nuclear envelopes (Fig. 15). Continuity of the endoplasmic reticulum with the nuclear envelope also is seen frequently. As in the elongated nuclei the rounded ones may show an attached bead-like structure (Fig. 8, arrow). Similar displaced, Feulgenpositive structures are seen in rounded nuclei undergoing division (Fig. 54, 72). Rounded nuclei vary in size from 1 to $3 \mu$.

Early stages of division-Rounded resting nuclei (Fig. 49) assume a ring-like form prior to division (Fig. 50, 68). The ring nuclei were often detected in the duplicated condition, sometimes with interzonal connections between the daughter threads (Fig. 50, 68, 51, 69, arrow).

As in the elongated nuclei the separating strands of ring nuclei may be free from one another or interlocked (Fig. 52, 70, 53, 71). Where the strands are interlocked (Fig. 53, 71) separation is effected only when at least one of the rings breaks (Fig. 54, 72). Distinct chromosomes are apparent only after separation of daughter rings (Fig. 54, 72).

Telophase-Telophasic transformations are rapid and daughter chromosomes are indistinct and become part of the resting nucleus. Transitional stages are seen in Fig. 55. The rounded nuclei so formed may remain in the resting stage till the end of the growth period or may assume the ring shape and divide again.

A semi-diagrammatic representation of the proposed scheme of vegetative nuclear division in Neurospora is shown in Fig. 73.
Discussion-A constant feature of the cytology of both wild and "clock" strains of Neurospora is the occurrence of elongated and rounded nuclei both of which can divide independently. Elongated nuclei in other fungi were reported by Lander (1934) in Arachnion album, Colson (1935) in Psalliota (Agaricus) campestris, and Carr and Olive (1958) in Sordaria. However, all these authors interpreted the elongated nuclei as migratory forms. As far as we know, dividing elongated nuclei have been recorded only in the deuteromycete, Verticillium (Roth and Brandt, 1964).

The longitudinal duplication of the nuclei in Neurospora has been observed and interpreted as a stage(s) of division by Dowding and Weijer (1962) and Weijer et al. (1963). The latter authors reported a filamentous phase at the beginning and end of division and a ring stage in the middle of each division. Dowding and Weijer (1962) observed that at the beginning of division the "coarse reticulum" of the nucleus forms a coiled protoplasmic thread which lies within the nuclear membrane and "as mitosis proceeds the nuclear membrane disappears and then the chromatin thread or ribbon unccils itself and lies free in the cytoplasm." In other words, these authors suggest that the "elongate structure" is merely a stage in the division cycle, instead of an interphase nucleus as well.

Our conclusions differ from those of Weijer et al. (1963) as follows: (1) Elongated nuclei have distinct nuclear envelopes (Fig. 16, 18) and, therefore, seem to be a distinct nuclear form and not just a stage in division subsequent to the disintegration of the nuclear envelope as suggested by Dowding and Weijer. (2) Both Dowding and Weijer (1962) and Weijer et al. (1963) describe the course of nuclear division as proceeding from a rounded nucleus through a thread-like stage and finally to the rounded form again. The present observations show that the rounded nuclei that migrate from the end of a period into the growing hyphae transform into elongated nuclei and retain the elongated form for approximately

Fig. 56-72. Interpretative diagrams of nuclear stages in Neurospora crassa. The numbers in parentheses refer to the photographic figures.-Fig. 56(10). Elongated nuclei in the initial growth phase. The slender interchromosomal strand with which a knob-like structure is attached to the nucleus is indicated by the letter "s." The arrow shows a dividing nucleus with stretched daughter strands and to its left is a dividing nucleus with daughter strands wrapped around each other.-Fig. 57(25). A spiral nucleus in which the direction of the spiral reverses.-Fig. 58 (26). A spiral nucleus immediately before division showing the beginning of despiralization.-Fig. 59(27). An elongated nucleus showing duplication of the chromatinic strand at one end. The individual strands show bead-like structures.-Fig. 60(30). Despiralization of the strands.-Fig. 61(31). Separation of the strands without intertwining. Duplication proceeding from both ends.-Fig. 62(32). A later stage in the separation of the strands showing bead-like bodies.-Fig. 63(34). Separation of the strands with one of the central chromosomes displaced (arrow).-Fig. 64(35). A configuration in which one of the displaced chromosomes is a subterminal chromosome of the chain (arrow).-Fig. 65(36). Separation of the strands with nonparallel connections.-Fig. 66(38). The distinct chromosome stage showing seven chromosomes with the central one displaced.-Fig. 67(40). A chain of seven chromosomes with the terminal chromosome displaced. The chromosome at the lower end of the chain has a satellite-like structure (arrow).-Fig. 68(50). A ring stage with duplicated strands.-Fig. 69(51). Incomplete and complete ring nuclei.-Fig. 70(52). Duplicating ring nuclei, the daughter strands may or may not be interlocked.-Fig. 71(53). Duplicating ring nuclei showing interlocking.-Fig. 72(54). Separation of daughter strands of interlocked rings. Arrows indicate the displaced chromosomes of the rings. All figures $\times 4000$. 


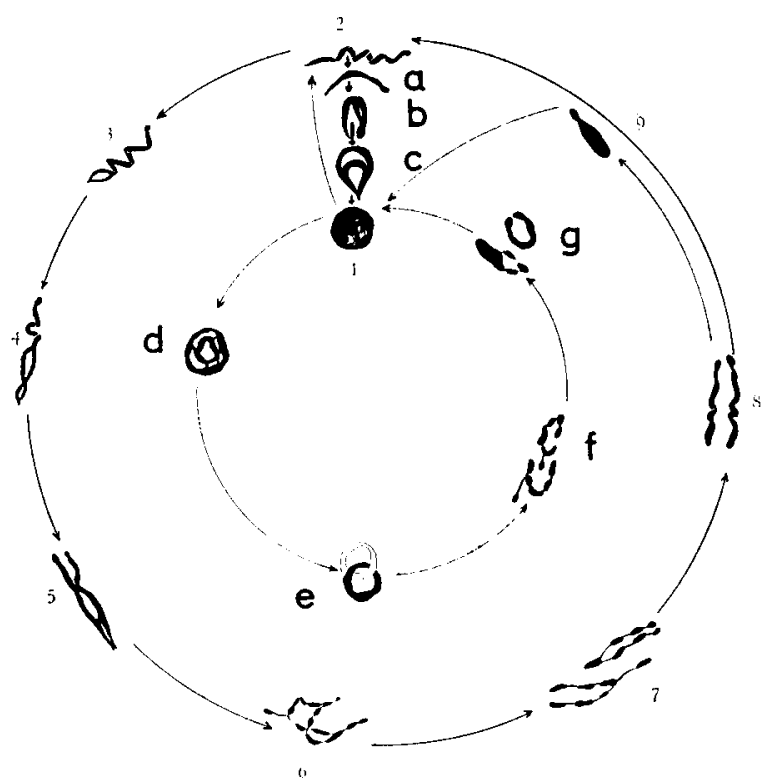

Fig. 73. Diagrammatic representation of vegetative nuclear division in Neurospora (the "clock" mutant). 1. Rounded resting nucleus (found in increasing proportions from 4-6 hr onwards in a growth period until it forms the predominant type of nucleus at the end of the period).-1-2. Conversion of the rounded resting nucleus to an elongated form at the beginning of the growth period.-2-8. Division stages in the elongated nuclear type.-8. Telophase of elongated nuclei. The elongated nuclei at this stage may undergo linear condensation and form rounded resting nuclei (8-9-1), or may pass on to the next division cycle without reverting to the rounded form (8-9-2), or may give rise to nuclei of various degrees of annularity $(2-a-b-c)$ or ring nuclei $(d)$. $-1-d-g$. Conversion of rounded nucleus to a ring nucleus and its division.

$4 \mathrm{hr}$. Division of the elongated nuclei without a change in their form is suggested by the great increase in the number of elongated forms during this phase, even when continued migration of nuclei to the growing hyphae was blocked by the removal of old mycelium after the new period has begun. In further support of this suggestion, the duration of division as calculated by Bakerspigel (1959) is $10 \mathrm{~min}$. (3) A number of forms that are transitional between elongated and rounded nuclei were seen beginning after $4 \mathrm{hr}$ and became especially numerous after the new growth period had developed for more than $8 \mathrm{hr}$. (4) Parallel stages of division, including the occurrence of discrete chromosomes, are noticed in the division of elongated and rounded nuclei (Fig. 40, 67, 42, $43,54,72)$.

Occurrence of compound chromosomes-One of the basic interpretations of the scheme of vegetative nuclear division presented here is the association of seven chromosomes to form a compound structure. The individual chromosomes are connected by slender, less darkly stained, inter- chromosomal strands. The presence of these strands during the chromosome stage as well as during the process of duplication and separation of the chromatin threads suggests that the seven chromosomes act as a single unit throughout division. Also the occurrence of unduplicated spiral elongated nuclei at the beginning of the division cycle, despiralization of the duplicated chromatinic strand, and the existence of daughter strands loosely wrapped about each other is reminiscent of the behavior of individual somatic chromosomes in the prophase of mitosis in some higher plants.

Instances of the occurrence of compound chromosomes in fungi, higher plants, and animals have been reported previously. McGinnis (1953) found thread-like structures connecting the chromosomes in the early stages of somatic division in the mycelium and spores of Puccinia graminis. In higher plants, strands that connect the chromosomes appear in the prophase of somatic mitosis in the angiosperm, Luzula (Brown, 1954). These strands connect the whole group of 12 chromosomes in metaphase and it is the chromosome chains that separate in anaphase. Formation of a compound chromosome occurs in Ascaris. Somatic mitosis is normal in Ascaris, but in meiosis, telomere-to-telomere connections result in the formation of a giant chromosome made up of many smaller chromosomes (White, 1936).

The nature and origin of interchromosomal strands are not known. Brown (1954) attempted to explain the origin of the connecting strands of chromosomes in Luzula, which appear only in the late prophase of somatic mitosis. Because the nucleolus disappears at the time of chromosome linkage, Brown (1954) suggested that the strands are derived from the nucleolus. The chromosome chains so formed in Luzula persist through anaphase, but the prochromosomes of the ensuing prophase are free and without connections. Since the chromosomes in Neurospora are linked and act as a unit throughout the division cycle, it is doubtful whether the strands have an extrachromosomal origin.

Significance of somatic compound chromosomesMazia (1961) has suggested that chromosomes are organized for attaching large numbers of genes to small numbers of centromeres for efficient handling. In Neurospora the presence of a displaced chromosome and separation of daughter chromosome chains suggest a single functional centromere, and the ever-present interchromosomal strands assure the functioning of the chromosome chain as a single unit throughout division. Also, it seems that in an organism like Neurospora, with pronounced cytoplasmic streaming, the organization of chromosomes into a unit would keep those of the same haploid complement together throughout the division cycle. 
Chromosome number-In several counts, the number of chromosomes was found to be seven. This corroborates all other previous reports except that of Somers et al. (1960), who recognized a tiny eighth chromosome in approximately one-fourth of their figures. Our experience shows that, even in the living hyphae, occasional breakage of interchromosomal strands, the consequent drift of separated chromosomes resulting from protoplasmic streaming, and the proximity of vagrant chromosomes to full haploid complements, account for deviation in the chromosome number from seven. The present estimate is based on the chromosome number in the most frequently found haploid complement (with interchromosomal strands) noticed in our preparations.

Chromosome morphology-The most detailed account of the morphology of the somatic chromosomes of Neurospora so far reported was contributed by Somers et al. (1960). According to them, there are two large chromosomes and five smaller ones. One of the larger chromosomes was said to have a satellite. However, we find that in elongated nuclei, in which the morphology of the chromosome is easily observed, the haploid complement consists of two large chromosomes, one of medium length, and four short ones. One of the terminal chromosomes usually stains more darkly than the remaining ones of the chain and is attached to the latter by a comparatively longer interchromosomal strand. The other terminal chromosome has a satellite-like body attached to it (Fiig. 40, 67, 43). Median constrictions also are seen in some of the other chromosomes. Variations in the length of the chromosome group affect the size and morphology of the individual chromosomes. An extreme case is seen in Fig. 44 where the terminal portions are stretched, thereby distorting the appearance of the terminal chromosome.

Interzonal connections-The interzonal connections noticed in the separating strands are similar in appearance to the connections reported in separating chromosomes at anaphase in some higher plants. Usually these connections are found between one or more chromosomes at anaphase, and Schrader (1953) has classified the various types observed by himself and others. According to him the connections arise from the pulling out of the chromosome sheath or pellicle, and variations among different organisms in the extent of connections arise from differences in the viscosity of the ensheathing material. However, in Luzula, it is not the chromosomes but the chromosome chains that separate, forming connections derived from the matrix (Brown, 1954). The interconnections in Neurospora also are between separating chromosome chains but we have no exact data as to their origin.
Comparison with previous descriptions of milosis in Neurospora-Bakerspigel (1959) reports the participation of a Feulgen-positive granule and an elongated central body (later called nucleolus) in the separation of the chromatin. The granule of Bakerspigel is considered to be a displaced chromosome in this report. The closest thing to the elongated central body of Bakerspigel seen in this study are the diffuse interzonal connections in Fig. 37. As explained before, these connections are interpreted as being extensions of matrical material.

The concept of the longitudinal split of the chromatin thread (Dowding and Weijer, 1962; Weijer et al., 1963; and Weijer, Koopmans and Weijer, 1965) is supported and extended by the observations presented in this study. In their latest paper Weijer et al. (1965) distinguish three division cycles in the mycelium of Neurospora crassa: Juvenile cycle, restricted to very fast growing mycelia; Maturation cycle I, occurring prior to the development of macroconidia and Maturation cycle II, coinciding with the formation of microconidia. The sphere-filament-ringfilament sequence they have suggested for the juvenile cycle naturally assumes that both filamentous and ring configurations are found in the same mitotic cycle. However, the evidence presented in this paper indicates the duplication of rounded and elongate nuclei without change of their form. Therefore, it is suggested that in division the elongated and rounded nuclei follow independent though parallel pathways. Also, according to Weijer et al. (1965) the starting point of Maturation cycle $I$ is considered to be a stage in the juvenile cycle ("horse-shoe phase") and that of the Maturation cycle II is apparently a stage ("monk-cap phase") in Maturation cycle I. Hence, these cycles are somewhat interconnected and again are not similar to the independent pathways of division suggested in this paper for elongated and rounded nuclei.

Ward and Ciurysek (1962) concluded that classical mitosis occurs in Neurospora. Their metaphase figures show deeply stained rounded or oval chromosomes, sometimes connected by less deeply stained material. In general, they resemble the condensed chromosomes of the separating daughter strands in Fig. 54, 72 of this report. However, their illustrations show chromosome groups where some of the chromosomes are fully separated from one another. It is suggested that the preservation of interchromosomal strands in the chromosome groups observed in the present study may result from the use of mycelial mats, thereby avoiding mechanical pressure on the hyphae in the course of preparation.

\section{LITERATURE CITED}

BAKERSPIGEL, A. 1959. The structure and manner of division of the nuclei in the vegetative mycelium of Neurospora crassa. Amer. J. Bot. 46: 180-190. 
Brown, S. W. 1954. Mitosis and meiosis in Luzula campestris DC. Univ. Calif. Publ. Bot. 27: 231-278.

Carr, A. J. H., ANd L. S. Olive. 1958. Genetics of Sordaria fimicola. II. Amer. J. Bot. 45: 142-150.

Colson, B. 1935. The cytology of the mushroom Psalliota campestris Quel. Ann. Bot. 49: 1-38.

Dowding, E. S., and J. WerJer. 1960. Mitosis in Neurospora. Nature 188: 338-339.

, AND - 1962 . Mitosis in Neurospora and Gelasinospora I. Genetica 32: 339-351.

Honowitz, N. H. 1947. Methionine synthesis in Neurospora. The isolation of cystathionine. J. Biol. Chem. 171: $255-264$.

Hrusfovetz, S. B. 1956. Cytological studies of Helminthosporium sativum. Can. J. Bot. 34: 321-327.

LANDER, C. A. 1934. The development of the fruiting body of Arachnion album. J. Elisha Mitchell Sci. Soc. 50: $275-282$.

LEUCHTEN BERGER, C. 1958. Quantitative determination of DNA in cells by Feulgen microspectrophotometry, p. 219-278. In J. F. Danielli [ed.], General cytochemical methods. Vol. I. Academic Press, New York.

LUFT, J. H. 1961. Improvements in epoxy resin embedding methods. J. Biophys. Biochem. Cytol. 9: 409-414.

Mazia, D. 1961. Mitosis and the physiology of cell division, p. 77-412. In J. Brachet and A. E. Mirsky [ed.], The cell. Vol. III. Academic Press, New York.

McGinnis, R. C. 1953. Cytological studies of chromosomes of rust fungi. I. The mitotic chromosomes of Puccinia graminis. Can. J. Bot. 31: 522-526.
Roth, J. N., AND W. H. Brandt. 1964. Nuclei in spores and mycelium of Verticillium. Phytopathology 54: 363-364.

Schrader, F. 1953. Mitosis. Columbia Univ. Press, New York.

Somers, C. E., R. P. Wagner, and T. C. Hsu. 1960. Mitosis in vegetative nuclei of Neurospora crassa. Genetics 45: 801-810.

Sussman, A. S., T. Durkee, and R. J. Lowry. 1965. A model for rhythmic and temperature-independent growth in "clock" mutants of Neurospora. Mycol. Mycopath. Appl. 25: 381-396.

, R. J. Lowny, ANd T. Durkee. 1964. Morphology and genetics of a periodic colonial mutant of Neurospora crassa. Amer. J. Bot. 51: 243-252.

VogeL, H. J. 1956. A convenient growth medium for Neurospora (Medium N). Microb. Genet. Bull. 3: $42-43$.

Ward, E. W. B., and K. W. Ciurysek. 1962. Somatic mitosis in Neurospora crassa. Amer. J. Bot. 49 393-399.

Werjer, J., A. Koopmans, And D. L. WeiJer. 1963. Karyokinesis in vivo of the migrating somatic nucleus of Neurospora and Gelasinospora species. Trans. N. Y. Acad. Sci., ser. II, 25: 846-854. , —— AND - 1965. Karyokinesis of somatic nuclei of Neurospora crassa III. The juvenile and maturation cycles (Feulgen and crystal violet staining). Can. J. Genet. Cytol. 7: 140-164.

White, M. J. D. 1936. The chromosome cycle of Ascaris megalocephala. Nature 137: 783.

Amer. J. Bot. 54(6): 748-754. 1967.

\title{
A PENNSYLVANIAN CALAMITEAN CONE WITH ELATERITES TRIFERENS SPORES ${ }^{1}$
}

\author{
Robert W. Baxter and Gilbert A. Leisman \\ Botany Department, University of Kansas, Lawrence \\ Biology Department, Kansas State Teachers College, Emporia
}

A $\quad$ B $\quad S \quad T \quad R \quad A \quad C \quad T$

In 1943 L. R. Wilson described some scattered elater-bearing spores found in a Middle Pennsylvanian coal ball from What Cheer, Iowa, as Elaterites triferens. The spores, averaging $60 \mu$ in diameter, with a trilete scar on the proximal surface and three conspicuous elaters attached to their distal surface, have now been found in a fragment of a cone from the same locality. The cone is similar to Calamostachys in having whorls of sporangiophores, each with four adaxial sporangia, and some secondary xylem at the nodes, but it possibly differs in lacking sterile bracts.

Pennsylvanian elater-bearing spores, Elaterites triferens, were first observed by Wilson (1943) in peel preparations of a coal ball collected at the What Cheer Clay Products Company at What Cheer, Iowa. The stratigraphic position of the

${ }^{1}$ Received for publication 8 August 1966.

This is a part of the senior author's general study of the American coal ball flora supported by National Science Foundation grant GB-4933.
What Cheer Coal is uncertain but is generally considered to be in the lower part of the Des Moines Series. The spores were found in "several loose masses" with more numerous detached elaters, but there were no recognizable sporangia or cone remains. The spores had obviously been shed from the sporangia prior to preservation and were mostly incomplete, lacking one or more elaters. Wilson's illustrations showed little detail; 